\title{
EFEITO AGUDO DO EXERCíCIO AQUÁTICO NA FUNÇÃO PULMONAR DURANTE A GESTAÇÃO
}

Daniela Bonfim Cortês ${ }^{1}$, Dionei Ramos ${ }^{3}$, Karina Cristina Fernandes ${ }^{1}$, Ana Paula Rodrigues Rocha ${ }^{1}$, Mariane Fátima da Silva Araujo ${ }^{1}$, Renata Marques David ${ }^{2}$, Cristina Elena Prado Teles Fregonesi ${ }^{3}$, Edna Maria do Carmo ${ }^{3}$.

1Discente do Programa de Graduação Lato Sensu em Fisioterapia da UNESP - Presidente Prudente - SP. ${ }^{2}$ Discente do Programa de Pós Graduação Strictu Senso em Fisioterapia da UNESP - Presidente Prudente - SP. ${ }^{3}$ Docente do Departamento de Fisioterapia da UNESP Presidente Prudente - SP. E-mail: daniela_bcortes@hotmail.com

\section{RESUMO}

A gravidez é um período de adaptação que envolve diversas modificações no organismo materno e entre elas podem ocorrer alterações na função pulmonar. O estudo teve como objetivo avaliar a função pulmonar na gestação, pela espirometria, antes e a pós da realização de uma sessão do protocolo aquático. Participaram deste estudo, gestantes em qualquer idade gestacional, com encaminhamento medico, gravidez única e sem complicações obstétricas. O protocolo foi realizado em piscina coberta e aquecida, com temperatura entre $30^{\circ} \mathrm{C}$ a $34 \circ \mathrm{C}$, com duração de 50 minutos. Antes e após as sessões, foram verificados os sinais vitais de freqüência cardíaca, pressão arterial, saturação de oxigênio e aplicação da escala de Borg. O protocolo foi dividido em fases: aquecimento, alongamento, fortalecimento muscular e relaxamento. As participantes foram submetidas à espirometria antes e após o protocolo. Conclusão: De acordo com os resultados encontrados pôde-se observar uma tendência positiva na função pulmonar, após a realização de um protocolo de exercício físico aquático. Ainda sugerere-se que o número da amostra seja aumentado para melhor análise dos dados.

Palavras-chave: Gestação, Imediato, Espirometria.

\section{INTRODUÇÃO}

A gestação é uma característica fisiológica na vida da mulher e suas funções reprodutivas requerem intensificação e adaptação dos processos metabólicos e fisiológicos do corpo de acordo com o desenvolvimento fetal ${ }^{1}$.Entretanto, devido às alterações fisiológicas que ocorrem nesse período, a gestante pode tornar-se susceptível a diferentes doenças pulmonares com altos riscos de morbidade e mortalidade ${ }^{2}$.

Segundo Rezende ${ }^{1}$, essas adaptações hormonais e anatômicas que ocorrem no sistema respiratório durante a gestação, interagem e afetam na função pulmonar. De acordo com Pereira e Krieger $^{2}$, a função pulmonar pode sofrer alterações nas vias aéreas, na caixa torácica e na unidade respiratória. Essas alterações podem estar presentes mesmo em mulheres saudáveis, o que inclui uma queda gradual da capacidade residual funcional (CRF), o volume de reserva expiratório (VRE) e um aumento correspondente na capacidade inspiratória $(\mathrm{Cl})^{3,4,5}$. 
As alterações da fisiologia pulmonar durante a gravidez podem ser mensuradas por técnicas espirométricas. A espirometria é considerada um exame complementar de maior utilidade ao fisiodiagnóstico para análise quantitativa e qualitativa da função ventilatória e baseiase na medida de volumes e fluxos, particularmente o expiratório ${ }^{6}$.

Há evidências na literatura de alterações na função pulmonar durante a gestação $7,8,9$. Entretanto, nenhum destes avaliou o efeito agudo após um protocolo de exercícios aquáticos. Portanto, diante do exposto, o estudo teve como objetivo avaliar a função pulmonar na gestação, pela espirometria, antes e após a realização de uma sessão do protocolo aquático.

\section{MATERIAL E MÉTODOS}

\section{Casuística}

Tratou-se de um estudo longitudinal e prospectivo. Participaram deste estudo, gestantes encaminhadas ao Centro de Estudos e Atendimentos em Fisioterapia e Reabilitação da Faculdade de Ciências e Tecnologia (CEAFIR) - FCT/ UNESP - Campus de Presidente Prudente que participavam do projeto de extensão "Fisioterapia Aquática na Gestação". Esse estudo fez parte do projeto de pesquisa "Programa de Avaliação e Intervenção de Fisioterapia Pré-natal da FCT/UNESP". As gestantes foram devidamente informadas sobre os procedimentos e objetivos deste estudo e após concordarem, assinaram o Termo de Consentimento Livre e Esclarecido (TCLE) aprovado no protocolo no 81/2011. Foram incluídas gestantes em qualquer idade gestacional, com encaminhamento medico, gravidez única e sem complicações obstétricas. Primeiramente foi realizado uma avaliação com dados de anamnese e exame físico e posteriormente foi submetida ao teste de função pulmonar. As gestantes foram avaliadas pela espirometria antes e após uma única sessão de exercícios físicos aquáticos.

\section{Prova de Função Pulmonar}

Para mensurar essa variável foi escolhido o teste de espirometria, realizado por meio de espirômetro fechado da marca MIR-Spirobank versão 3.6 acoplado a um microcomputador que utiliza para análise o softwearOcean\&WinSpiro para Windows versão $1.04 \mathrm{~A}$, pertencente ao CEAFIR da FCT/UNESP. Os testes espirométricos foram obtidos a partir da manobra de Capacidade Vital Forçada (CVF), cujo procedimento segue descrito abaixo: - com o clipe nasal e a orientação para o participante respirar normalmente pelo bucal do aparelho, o indivíduo recebeu o comando para inspirar profundamente até atingir sua capacidade pulmonar total e logo em seguida expirar 
todo o ar o mais rápido possível, sustentando a expiração até que o observador ordenasse a interrupção.

O teste foi considerado válido de acordo com as Diretrizes de espirometria para testes de função pulmonar, ou seja: o valor de retroextrapolação, não passou de $5 \%$ da CVF ou $150 \mathrm{ml}$; a inspiração até a CPT antes da expiração forçada não foi muito rápida; a pausa pós-inspiratória não excedeu 3s; o início do teste foi abrupto e sem hesitação; a duração da expiração forçada foi de no mínimo 6s; o platô evidente na curva volume-tempo ocorreu por pelo menos $2 \mathrm{~s}$; cada indivíduo realizou três curvas aceitáveis, isto é, teve o PFE dentro de $0.5 \mathrm{l} / \mathrm{s}$ do maior PFE obtido em manobras prévias e duas reprodutíveis, isto é, os dois maiores valores de VEF 1 e CVF diferiram menos de $0.150 \mathrm{l}$; a CVF selecionada foi a de maior valor dentre as curvas com valores de PFE situados dentro dos critérios de aceitação ${ }^{10}$.

\section{Protocolo Aquático}

O programa de exercícios físicos aquáticos foi realizado em piscina coberta e aquecida, com temperatura entre $30^{\circ} \mathrm{C}$ a $34^{\circ} \mathrm{C}$, duas vezes por semana com duração de 50 minutos. Antes e após o protocolo, foram verificados os sinais vitais de freqüência cardíaca, pressão arterial, saturação de oxigênio como condição clínica para realizar a sessão. O protocolo foi dividido em fases: 1) 10 minutos de aquecimento (ex. caminhada ao redor da piscina, pedalando no espaguete); 2) 10 minutos de alongamento de membros superiores e inferiores, região cervical e tronco;3) 20 minutos (2 séries de 10 a 15 repetições) de exercício para membros superiores e membros inferiores com caneleiras e halteres e mobilizações articulares; 4) 10 minutos de relaxamento, deitadas sobre o espaguete e utilizando colete cervical ao som de música suave.

\section{RESULTADOS}

Foram avaliadas 16 gestantes, com características descrita nas tabelas 1 e 2 .

Tabela 1. Características da amostra de gestantes.

\begin{tabular}{|l|l|}
\hline Idade (anos) & $26 \pm 6$ \\
IMC (Kg/ $\mathrm{m}^{2}$ ) & $25 \pm 3$ \\
Período Gestacional (semanas) & $24 \pm 4$ \\
\hline
\end{tabular}

* Os dados foram descritos em média e desvio-padrão.

Tabela 2. Dados da espirometria antes e após os exercícios aquáticos. 


\begin{tabular}{|c|c|c|c|}
\hline Índices Espirométricos & Pré-exercício & Pós-exercício & p-Valor \\
\hline CVF (L) & $3,34 \pm 0,38$ & $3,35 \pm 0,35$ & $*$ \\
\hline CVF(\% prev) & $92 \pm 10$ & $92 \pm 10$ & $*$ \\
\hline VEF1 (L) & $2,84 \pm 0,27$ & $2,85 \pm 0,26$ & $*$ \\
\hline VEF1 (\% prev) & $91 \pm 8$ & $91 \pm 8$ & $*$ \\
\hline VEF1/CVF & $0,85 \pm 0,04$ & $0,85 \pm 0,03$ & $*$ \\
\hline PFE (L/s) & $7,35 \pm 0,68$ & $7,25 \pm 0,94$ & $*$ \\
\hline PFE (\% prev) & $100 \pm 12$ & $97 \pm 13$ & $*$ \\
\hline $\mathrm{FEF}_{25-75 \%}(\mathrm{~L} / \mathrm{s})$ & $3,52 \pm 0,77$ & $3,43 \pm 0,63$ & $*$ \\
\hline $\mathrm{FEF}_{25-75 \%}(\%$ prev $)$ & $96 \pm 17$ & $95 \pm 16$ & $*$ \\
\hline \multicolumn{4}{|c|}{$\begin{array}{l}\text { CVF: capacidade vital forçada; VEF1: volume expiratório forçado no primeiro segundo; PFE: pico de fluxo expiratório; } \\
\mathrm{FEF}_{25-75 \%} \text { : Fluxo Expiratório Forçado } 25-75 \% \text {; (\% prev): porcentagem do valor previsto; (L): litros; (L/s): litros por segundo. } \\
\text { * valor estatístico não significativo }>>0,05 \text {. }\end{array}$} \\
\hline
\end{tabular}

Tabela 3. Dados espirométricos individual: antes e após os exercícios aquáticos.

\begin{tabular}{|c|c|c|c|c|c|c|c|c|c|c|}
\hline \multirow[b]{2}{*}{ № } & \multicolumn{2}{|c|}{ CVF\% do previsto } & \multicolumn{2}{|c|}{$\begin{array}{l}\text { VEF1\% do } \\
\text { previsto }\end{array}$} & \multicolumn{2}{|c|}{ VEF1/CVF } & \multicolumn{2}{|c|}{$\begin{array}{l}\text { PEF\% do } \\
\text { previsto }\end{array}$} & \multicolumn{2}{|c|}{$\begin{array}{c}\text { FEF25-75\% do } \\
\text { previsto }\end{array}$} \\
\hline & Pré-EA & Pós-EA & Pré-EA & Pós-EA & $\begin{array}{l}\text { Pré- } \\
\text { EA }\end{array}$ & $\begin{array}{l}\text { Pós- } \\
\text { EA }\end{array}$ & Pré-EA & Pós-EA & Pré-EA & Pós-EA \\
\hline 1 & 93 & 93 & 91 & 92 & 0,88 & 0,90 & 117 & 105 & 95 & 90 \\
\hline 2 & 107 & 105 & 102 & 100 & 0,81 & 0,81 & 92 & 92 & 89 & 85 \\
\hline 3 & 108 & 105 & 98 & 96,5 & 0,77 & 0,77 & 100,58 & 91 & 79 & 83 \\
\hline 4 & 95,9 & 95 & 93,9 & 91,9 & 0,85 & 0,84 & 98 & 94,7 & 84 & 77 \\
\hline 5 & 72 & 74 & 77 & 77 & 0,89 & 0,87 & 87 & 94 & 90 & 88 \\
\hline 6 & 88 & 96 & 83 & 92 & 0,80 & 0,81 & 92 & 97 & 72 & 84 \\
\hline 7 & 81 & 82 & 82 & 82 & 0,84 & 0,84 & 103 & 107 & 90 & 85 \\
\hline 8 & 88 & 87 & 94 & 93 & 0,89 & 0,89 & 100 & 99 & 110 & 111 \\
\hline 9 & 93 & 81 & 88 & 83 & 0,87 & 0,85 & 117 & 96 & 104 & 100 \\
\hline 10 & 94 & 94 & 94 & 92 & 0,91 & 0,89 & 103 & 90 & 98 & 96 \\
\hline 11 & 109 & 110 & 108 & 110 & 0,84 & 0,85 & 99 & 89 & 112 & 127 \\
\hline 12 & 96 & 91 & 93 & 87 & 0,82 & 0,81 & 76 & 71 & 87 & 78 \\
\hline 13 & 89 & 94 & 92 & 95 & 0,94 & 0,92 & 127 & 133 & 141 & 125 \\
\hline 14 & 89 & 91 & 93 & 95 & 0,86 & 0,86 & 94 & 98 & 117 & 115 \\
\hline 15 & 91 & 92 & 88 & 92 & 0,82 & 0,84 & 102 & 107 & 87 & 98 \\
\hline 16 & 78 & 78 & 78 & 78 & 0,86 & 0,86 & 94 & 94 & 79 & 79 \\
\hline Média & 91,99 & 91,75 & 90,93 & 91,03 & 0,85 & 0,85 & 100,10 & 97,36 & 95,88 & 95,06 \\
\hline $\begin{array}{l}\text { Desvio- } \\
\text { padrão }\end{array}$ & 10,25 & 9,88 & 8,26 & 8,32 & 0,04 & 0,04 & 12,33 & 12,75 & 17,45 & 16,38 \\
\hline
\end{tabular}

Não foi observada diferença estatística significante em nenhuma variável espirométrica estudada, quando comparados os momentos antes e após uma única sessão de exercício aquático $(p>0,05)$. Entretanto, das 16 gestantes estudadas, 3 destas, aumentaram todos os valores dos 
índices espirométricos pós exercício aquático conforme o destaque na tabela 3 , o que sugere uma discreta melhora na função pulmonar.

\section{DISCUSSÃO}

A espirometria é considerada o melhor método para a mensuração da função pulmonar. 0 fluxo expiratório corresponde à quantidade de ar expelida em milissegundos, medida em litros por minutos, durante uma expiração forçada. ${ }^{20}$

Observou-se em um estudo, com gestantes entre 32 a 36 semanas com média de idade de 20,67 $\pm 2,52$ anos, que houve diminuição na capacidade pulmonar sendo considerável, de acordo com a idade, como fisiológico e não patológico ${ }^{9}$. No presente estudo não se aplica avaliar se existe ou não diminuição da capacidade pulmonar em relação aos trimestres gestacionais, porém analisando o protocolo observamos melhora percentual.

Para Redivo, mudanças na função pulmonar são provavelmente pequenas demais pra explicar o surgimento da dispnéia, sintoma comum durante a gestação, mas as alterações da caixa torácica poderiam influenciar o surgimento dos mesmos, o mesmo autor relata diminuição dos índices espirométricos durante o terceiro trimestre gestacional, porém, não o considera patológico, e sim, restritivo ${ }^{9}$.

No presente estudo quase a metade das gestantes apresentaram um aumento nos valores de CFV após o exercício aquático. E de acordo com Araújo ${ }^{11}$ o exercício aquático melhora a capacidade funcional de indivíduos vulneráveis, como DPOC's, portanto, pode ser que durante a gestação também ocorra o mesmo.

Algumas limitações do estudo precisam ser apresentadas: Algumas gestantes apresentavam medo durante a avaliação da função pulmonar mas ainda assim o exame era realizado, outras não conseguiam realizar no dia agendado, então a sessão aquática e o exame de avaliação da função pulmonar eram cancelados e remarcados para outro dia.

Poucos estudos tem investigado o efeito de exercícios na função pulmonar em gestantes. Portanto, pretende-se a médio e longo prazo aumento do número de avaliações para verificação da relação do efeito de exercício aquático na gestação.

\section{CONCLUSÃO}


De acordo com os resultados encontrados pôde-se observar uma tendência positiva na função pulmonar, após a realização de um protocolo de exercício físico aquático. Ainda sugererese que o número da amostra seja aumentado para melhor análise dos dados.

\section{REFERÊNCIAS}

1. De Rezende J. Obstetrícia. 10. ed. Rio de Janeiro: Guanabara, 2005.

2. Pereira A, Krieger BP. Clin Chest Med. 25a Ed. 2004; 299-310.

3. Cugell D, Frank N, Gaensler E. Pulmonary function in pregnancy. American Review of tuberculosis 1953; 67: 568- 597.

4. Lehmann V, Fabel H. (Investigation of respiratory function in pregnancy. I. Vital Capacity) [Author's Translation]. Z GeburtshilfePerinatol 1973;177(6):387-396.

5. Mcauliffe F, Kametas N, Costello J, Rafferty GF, Greenough A, Nicolaides K. Respiratory function in singleton and twin pregnancy. $\mathrm{Br} J$ ObstetGynaecol 2002;109:765-769. http://dx.doi.org/10.1111/j.1471-0528.2002.01515.x

6. Miller, MR et al. General considerations for lung function testing. EurRespi J 2005; 26(1):153161. http://dx.doi.org/10.1183/09031936.05.00034505

7. Viana MCC. Análise da função pulmonar em gestantes de alto risco em uma maternidade pública do estado do ceará [dissertação]. Fortaleza: Universidade Estadual do Ceará; 2008.

8. Neppelenbroek GA, Gadelha PS, Mauad Filho F, Costa AG, Gallarreta FMP, Carvalho Filho OS, et al. Aspectos fisiológicos e diagnósticos das alterações pulmonares durante a gestação/ Physiologicalanddiagnosticaspectsofpulmonarychanges in pregnancy. Femina 2006; 559-563.

9. Redivo MB, Vinadé IA, Soares MG, Kock QS. Avaliação da função pulmonar em gestantes no período gestacional entre 28 a 36 semanas. 2007. (Supl1) p. 6-7.

10. Pereira CAC. Diretriz de espirometria. J Pneumol Out 2002; 28(Supl 3):1s-82s.

11. Araujo ZTS. Exercício físico de baixa intensidade na DPOC: Comparação dos programas solo e aquático. 2009. [Tese] Universidade Federal do Rio Grande do Norte. Natal, RN. 